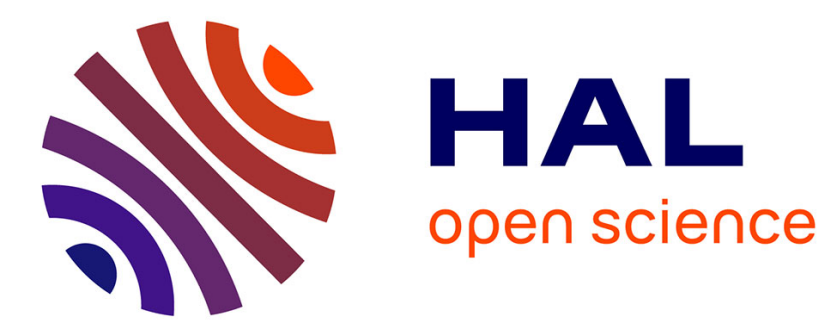

\title{
On the centre of mass motion in human walking
} Justin Carpentier, Mehdi Benallegue, Jean-Paul Laumond

\section{To cite this version:}

Justin Carpentier, Mehdi Benallegue, Jean-Paul Laumond. On the centre of mass motion in human walking. International Journal of Automation and Computing, 2017, 14 (5), pp.542-551. hal$01377361 \mathrm{v} 2$

\section{HAL Id: hal-01377361 \\ https://hal.science/hal-01377361v2}

Submitted on 15 May 2017

HAL is a multi-disciplinary open access archive for the deposit and dissemination of scientific research documents, whether they are published or not. The documents may come from teaching and research institutions in France or abroad, or from public or private research centers.
L'archive ouverte pluridisciplinaire HAL, est destinée au dépôt et à la diffusion de documents scientifiques de niveau recherche, publiés ou non, émanant des établissements d'enseignement et de recherche français ou étrangers, des laboratoires publics ou privés.

\section{다(1)(2)}

Distributed under a Creative Commons Attribution - ShareAlike| 4.0 International 


\title{
On the centre of mass motion in human walking
}

\author{
Justin CARPENTIER ${ }^{1} \quad$ Mehdi BENALLEGUE ${ }^{2} \quad$ Jean-Paul LAUMOND $^{1}$ \\ ${ }^{1}$ LAAS-CNRS, University of Toulouse, CNRS, Toulouse, France \\ ${ }^{2}$ Humanoids Research Group, National Institute of Advanced Industrial Science and Technology, Tsukuba, Japan
}

\begin{abstract}
The center of mass (CoM) is a key descriptor in the understanding and the analysis of bipedal locomotion. Some approaches are based on the premise that humans minimize the CoM vertical displacement. Other approaches express walking dynamics through the inverted pendulum model. Such approaches are contradictory in that they lead to two conflicting patterns to express the CoM motion: straight line segments for the first approaches and arcs of a circle for the second ones.

In this paper we show that CoM motion is a trade-off between both patterns. Specifically CoM follows a "curtate cycloid", which is the curve described by a point rigidly attached to a wheel rolling on a flat surface. We demonstrate that all the three parameters defining a curtate cycloid only depend on the height of the subjects.
\end{abstract}

Keywords: human locomotion, analytical model, centre of mass, locomotion signature, synergies

\section{Introduction}

Walking aims at transporting the body from a placement on the ground to another one. Two parameters of position and one parameter of orientation define the body placement. Human body motions originate in the motor space made of more than 600 muscles. Walking can then be viewed as a mathematical mapping from the very high dimensional body space into the 3-dimensional placement one. This makes walking a complex process for a simple-objective task ${ }^{[1]}$. Such complexity remains a challenge for many disciplines in life sciences (biomechanics, neurophysiology, medicine, physical therapy) and recently for computer engineering and robotics with the emergence of humanoid robots ${ }^{1}$. Most research approaches explore complexity reduction principles. For example, six major determinants of gait have been identified ${ }^{[4,5]}$ as critical features to address walking kinematics. The introduction of gait determinants have been mainly motivated by the minimization of CoM vertical displacement. On the other hand, a popular model to model walking dynamics is the inverted pendulum ${ }^{[6]}$. Inverted pendulum gives a rough approximation of the motion of the walker center of mass (CoM) via a sequence of arcs of a circle. The geometry of CoM motion induced by both perspectives are incompatible: minimizing the CoM vertical displacement tends to a straight line motion, which is not converging towards a motion made of a sequence of arcs of a circle. The contradiction of both theories is deeply explored in ${ }^{[7]}$. It is shown that both underlying premises are limited and it is proposed to focus on mechanical work rather than the kinematics or forces of gait.

Nevertheless, in any case, the estimation of CoM motion plays a central role in the study of human walking. It represents a descriptor of motion relevant in both kinematic and dynamic point of view and may allow validating

\footnotetext{
Manuscript received January 9th, 2017

${ }^{1}$ Beyond walking studies, the field of biomechanics of human motion is evolving rapidly towards multidisciplinary research $[2,3]$
}

or invalidating theories of human walking. However, reconstructing the position of CoM is not a straightforward process, since it is not rigidly linked to any limb of the body. For instance, in ${ }^{[8]}$, it is shown that CoM moves differently from the motion of the pelvis. The importance of stance-limb behavior in determining the trajectory of CoM during walking and running is explored in ${ }^{[9]}$. The path followed by CoM when walking on a treadmill has an upward concave figure-of-eight shape which is described in [10].

CoM position and motion estimation are addressed by modern techniques of motion capture. The human body CoM depends on various parameters, which are classically reduced to articular angles and limb mass distribution. Body segments are considered as rigid bodies. In vision-based motion capture, body segments are equipped with markers. Their 3D positions are captured by vision. Articular angles are deduced from the position of body parts ${ }^{[11,12]}$. CoM is then computed from standard anthropomorphic mass distribution of body parts ${ }^{[13]}$. A second popular approach is to estimate CoM position and motion directly from force platforms ${ }^{[14,15,16]}$. Such platforms measure the interaction forces and moments of the body with the environment. Forces are at the origin of the CoM accelerations while moments are related to CoM position by the mean of the so-called central axis of the contact wrench ${ }^{[3]}$.

It remains that both kinematics-based and dynamics-based approaches of CoM estimation are subject to a lot of inaccuracy sources. In ${ }^{[17]}$ we recently proposed a theoretical study about the observability of the center of mass position using motion capture and force platforms. We showed that the accuracy domain of each measurement can be easily described through a spectral analysis. We then introduced a new approach based on complementary filtering to estimate the CoM position with increased accuracy.

Based on this new CoM estimation algorithm, the present study explores the geometric shape of the CoM path when 


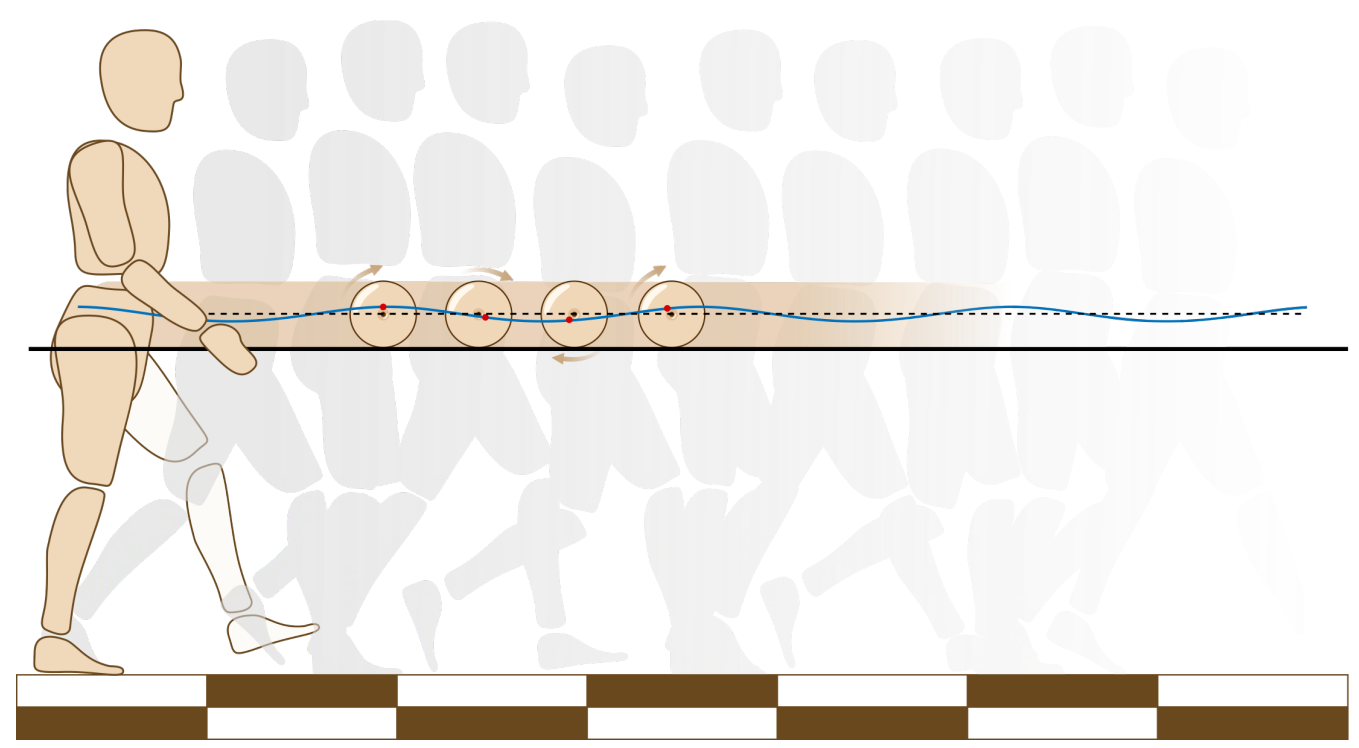

Figure 1: Illustration of the CoM trajectory in the sagittal plane during human walking. The CoM trajectory has a cycloidal pattern, described by a point on a wheel rolling at constant velocity on a flat surface.

walking. It is shown that CoM follows a "curtate cycloid" in the sagittal plane, generated by a virtual wheel whose parameters constitute original invariants of bipedal walking and illustrated on Fig. 1.

\section{Material and methods}

\subsection{Participants}

Twelve healthy male (age: $24.2 \pm 2.3 \mathrm{yr}$, height: $1.74 \pm$ $0.04 \mathrm{~m}$, mass: $71.0 \pm 8.9 \mathrm{~kg}$ ) and four female (age: $24.3 \pm 3.3$ yr, height: $1.71 \pm 0.04 \mathrm{~m}$, mass: $53.4 \pm 8.9 \mathrm{~kg}$ ) subjects volunteered for this investigation. The experiments were conducted in accordance with the standards of the Declaration of Helsinki (revision 2013), with formal approval of the ethics evaluation by the ethic committee of INSERM (IRB00003888, Opinion number 13-124) of the National Institute of Health and Medical Research, INSERM, Paris, France (IORG0003254, FWA00005831).

\subsection{Data acquisition}

The experiment room (dimension $6 \times 20 \mathrm{~m}$ ) was equipped with 12 infrared cameras sampling at $200 \mathrm{~Hz}$ (Vicon, Oxford Metrics, Oxford, UK) and recording 43 reflective markers placed on the whole body of the (see Fig. 2). Markers set is based on Wu recommendations and approved by the International Society of Biomechanics ${ }^{[11,12]}$. Two force plates (AMTI, Watertown, MA, USA) embedded into the floor were used to record ground reaction forces and ground reaction moments at $1000 \mathrm{~Hz}$.

\subsection{Experimental protocol}

Participants were instructed to walk barefoot in straight line at their normal walking speed (see Fig. 2). The walking distance was about $8 \pm 1 \mathrm{~m}$. At $4 \mathrm{~m}$ from the starting point, the subjects had to walk on the two consecutive force platforms. For each subject, 10 valid trials were recorded.
A trial was considered valid as soon as the stance foot was completely located on the force plates, allowing the full measurement of the external forces and wrenches.

\subsection{Center of mass reconstruction}

To fully reconstruct the center of mass position, we used an accurate estimator recently introduced by the authors and described in ${ }^{[17]}$. Improving CoM reconstruction methods currently used in Biomechanics [18, 19], this approach efficiently merges three different inputs: the external forces, external wrenches and the center of mass position computed from the marker positions and anthropomorphic tables ${ }^{[20]}$. All those signals carry noises and errors, but with different frequency bandwidths for each signal. Those measurements are then merged together according to their respective bandwidth accuracy thanks to a complementary filtering approach. As output, we obtained an estimation of the center of mass position which tends to be free of bias compared to previous measurement methods. This produces a more accurate estimate of the real CoM position, which is also consistent with respect to the external forces. This method offers the ability to both operate in online and off-line mode, thanks to optimization and averaging of forward and backward passes, resulting in a zero-delay output signal. We used this second mode to estimate the CoM trajectory.

\subsection{The curtate cycloid}

A cycloid is a curve corresponding to the path followed by a point $c$ attached to the radius of a wheel rolling without slipping on a plane surface. This curve is defined in the sagittal plane by the parametric cartesian equation:

$$
\begin{aligned}
x & =R \theta-r \sin (\theta) \\
z & =z_{0}-r \cos (\theta)
\end{aligned}
$$




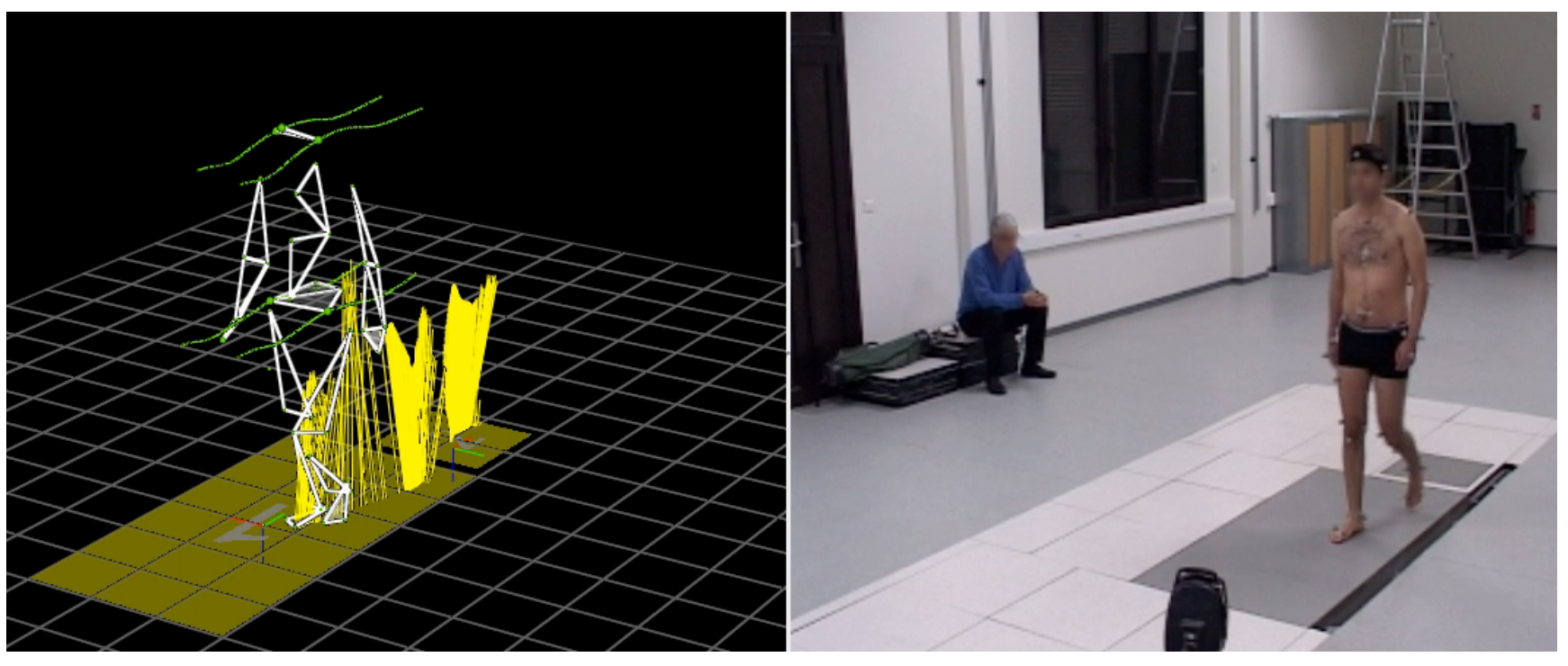

Figure 2: Capture of the experiment room during the acquisition session. A male subject was instructed to walk barefoot in straight line at his comfort walking speed on two force platforms. Two force plates are firmly embedded in the floor and allows the reconstruction of the segmentation of the walking pattern.

where $\theta$ is the angle between the radius of the wheel and the vertical direction, $R$ is the radius of the wheel, $r$ is the distance of the point $c$ to the wheel center and $z_{0}$ is the altitude of the wheel center. $x$ and $z$ are the coordinates of $c$ regarding the forward and vertical spatial axis. All in all, the cycloid is described by a set of 3 parameters denoted by $\boldsymbol{p}=\left[R, r, z_{0}\right]$ and one variable $\theta$ which evolves according to time. The first time derivative of the trajectory $\theta$ corresponds to the angular velocity $\omega$ of the cycloid. We may distinguish three cases which are illustrated by the Fig. 3. In the following, we are interested by the curtate cycloid. This curve has the property of being cyclic and asymmetric, similar to CoM trajectories for which the convex lower part of the cycle is longer than the concave higher part.

\subsection{Segmentation of the gait}

The use of two synchronized force plates enables us to efficiently and precisely segment the walking motion into single and double support phases.

\subsection{Fitting protocol}

The fitting process is based on numerical optimization. It is set up as a nonlinear least-square problem, where we try to minimize the distance between the CoM trajectory (the measurement) and the cycloidal model composed of three parameters $\boldsymbol{p}=\left[R, r, z_{0}\right]$ and one variable $\theta$ which evolves according to time. The fitting problem is written as:

$$
\min _{\boldsymbol{p}, \theta} \sum_{k=1}^{N}\left\|\boldsymbol{y}_{\text {mes }}^{k}-f_{\text {model }}\left(\theta^{k}, \boldsymbol{p}\right)\right\|_{2}^{2}
$$

where $f_{\text {model }}$ corresponds to the parametric models exposed in Eq. (2) and $\boldsymbol{y}_{\text {mes }}^{k}$ is the $k^{\text {th }}$ sample measurement of the CoM trajectory in the sagittal plane.

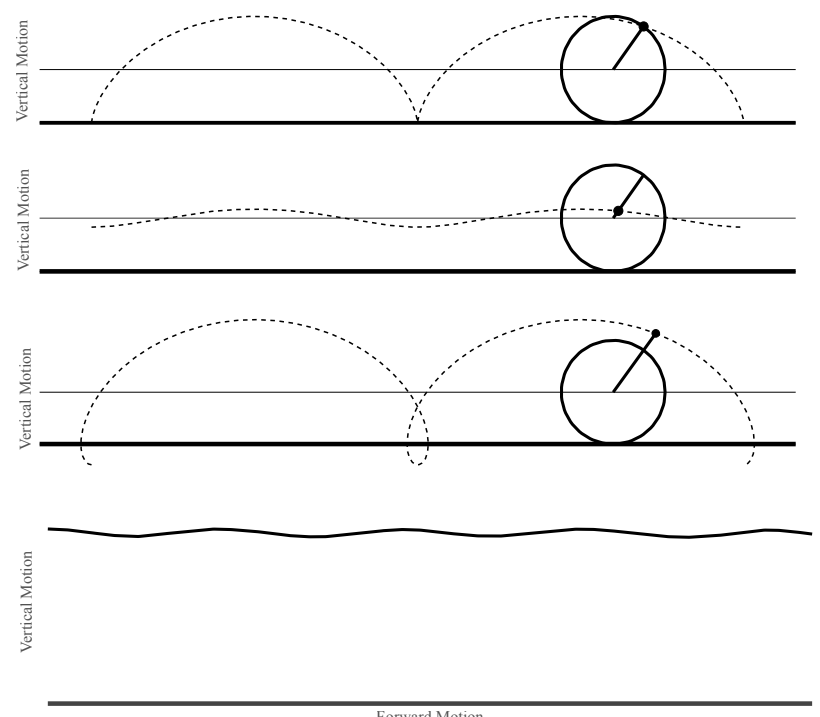

Figure 3: Illustration of the three types of cycloid. From top to bottom: normal cycloid, curtate cycloid and prolate cycloid. The last plot corresponds to the CoM trajectory in the sagittal plane. Its shape is very similar to the curtate cycloid.

This problem is efficiently solved with standard nonlinear least-square solvers. In this study we use the function lsqnonlin provided with MATLAB, The MathWorks, Inc., Natick, Massachusetts, United States.

Even though the natural walking is considered as a cyclic process, both the amplitude of the CoM trajectory and the cycle duration vary slightly even between two consecutive steps. To overcome those natural fluctuations, the data concerning each subject is made of a collection of ten single 


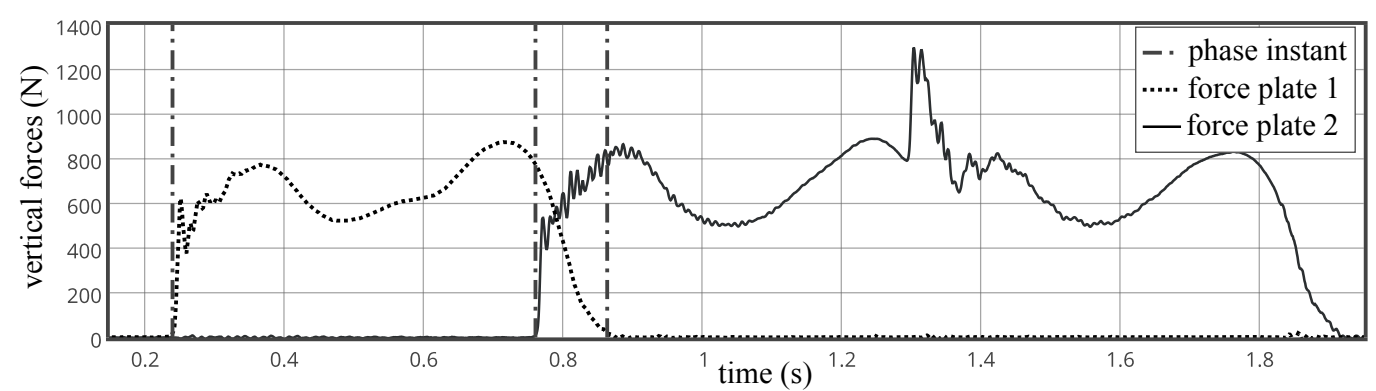

(a) Segmentation of the gait into single and double support phases. The two force plates allows an efficient detection of initial and final contact instant of the gait.

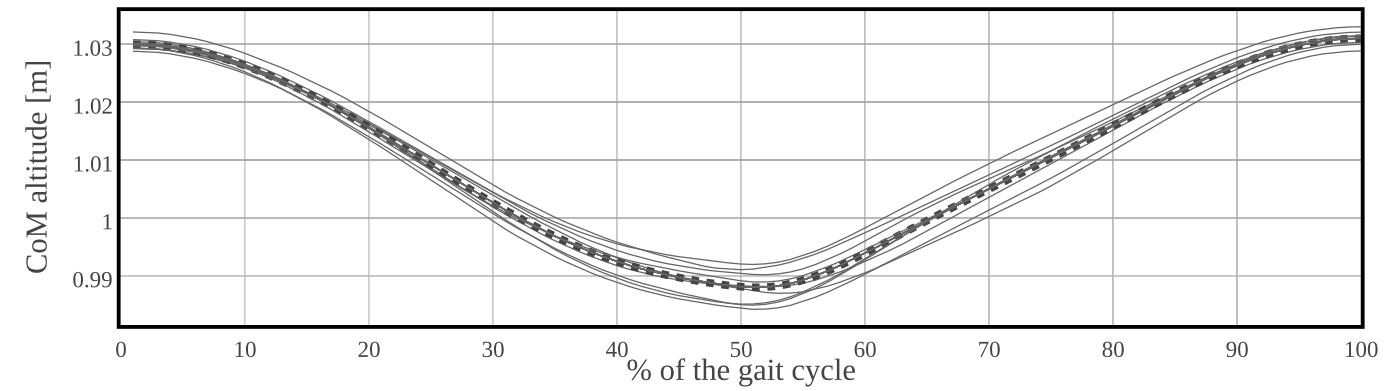

(b) Illustration of the variability of the CoM on the vertical direction during one single step. The solid curves represent the estimated trajectory of the CoM according to the percentage of the gait cycle and the dashed curve is the mean of all those trajectories.

Figure 4: Illustrations of the segmentation of the gait (a) and of the variability of the CoM during one single step (b).

steps. The standard intra-subject deviation is presented in Fig. 4b. Therefore, to overcome those natural fluctuations, we chose to operate the fitting procedure only for one step composed of a single support and double support phases. This choice allows to reach precisions finer than the intra-subject variability, and even to study this variability in terms of few parameters as we present later in this paper. In the following, we call this interval on which the optimization operates the fitting interval.

From the optimization result, we finally fit a polynomial of degree 1 to the trajectory $\theta$ according to time. The monomial of degree 1 finally corresponds to the angular velocity $\omega$ of the wheel. With this model, the equation $\theta$ is then given by:

$$
\theta(t)=\theta_{0}+\omega t
$$

\section{Results}

This section is organized as follows: we first present the results of the fitting process, we then expose the correlations between the fitting parameters and the height of the subjects. We conclude this section by showing the extraction of the temporal segmentation from the data of this model.

\subsection{Fitting of the model}

Hereinafter, we start by presenting the example of the fitting of one subject. Subsequently, we show statistical data about the quality of the reconstruction of all the subjects.
Fig. 5a shows both the result of the fitting for one fitting interval which corresponds to a full step composed of one single support and one double support. We see that the fitting is able to closely follow the trajectory of the CoM and to reproduce its asymmetry.

The error between the reconstructed CoM and the fitted trajectory is displayed in Fig. 5b on the forward and vertical motion axes. We can observe that the fitting error is lower than $1.5 \mathrm{~mm}$ on the entire duration of the cycle.

This level of fitting quality does not vary a lot among subjects. Fig. 6 shows the mean and the standard deviation of the reconstruction error for all subjects. In general, the mean reconstruction is less $3.5 \mathrm{~mm}$ with maximal standard deviations of $1 \mathrm{~mm}$. At this stage, it is worth to notice that for numerous subjects the mean error is less than $1 \mathrm{~mm}$ with very low standard deviation, less than $0.5 \mathrm{~mm}$.

This fitting quality allows to study human walking trajectories with a reduced number of parameters. One example is presented hereafter where we study the correlations that lie between the trajectory parameters and the height of the subjects.

\subsection{Link between model parameters and the subject's height}

From one step to another, the found fitting parameters are different due to the variability of the gait cycle. Nevertheless, the variation of these parameters is very small and may even be characteristic of each subject. But to properly support this claim, a higher number of subjects is necessary.

The following results study how these parameters 


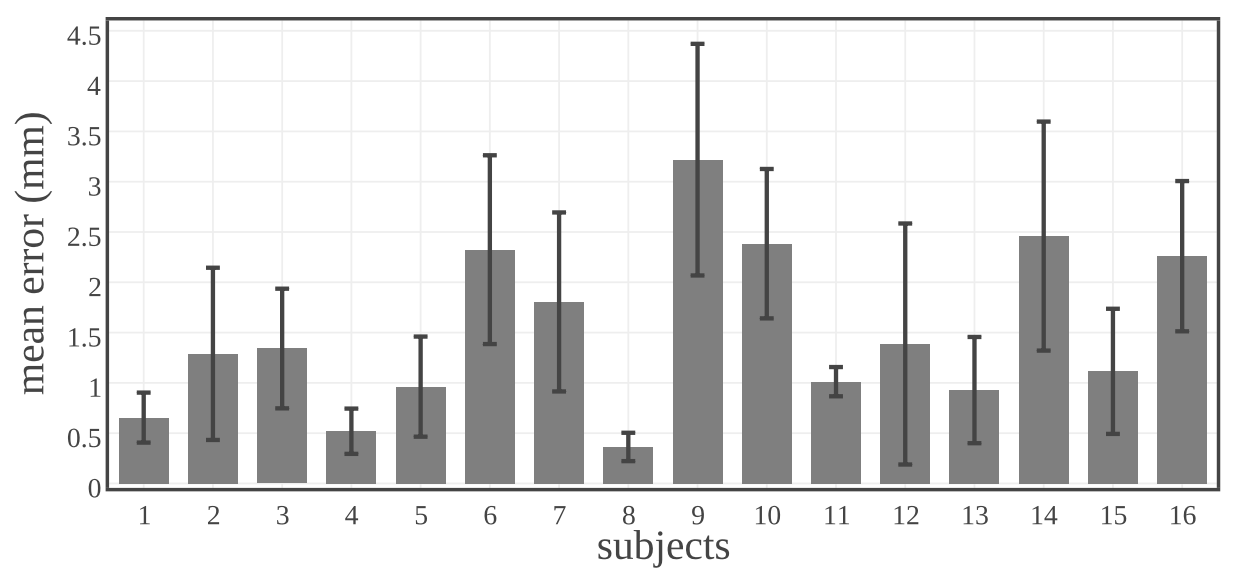

Figure 6: Mean and standard deviation of the reconstruction error for each subject. The mean reconstruction for all the subjects remains below $3.5 \mathrm{~mm}$ with a maximal standard deviation of $1.5 \mathrm{~mm}$.

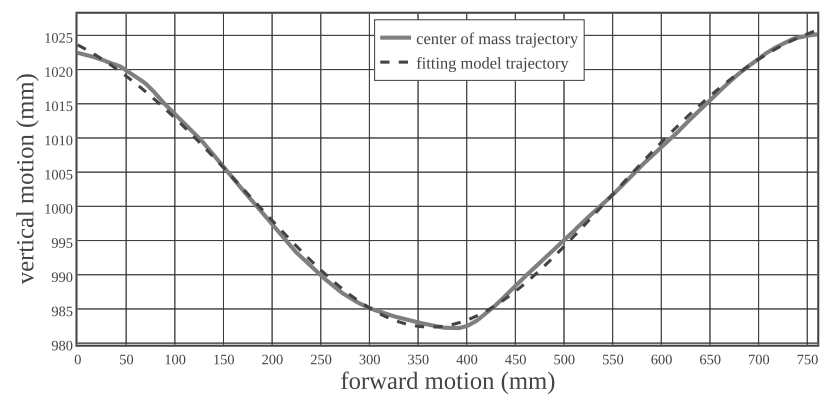

(a) Center of mass trajectory fitted with the proposed model The fitting closely follows the CoM trajectory and reproduces its asymmetry. Notice that, for a better reading, the axis coordinates are rescaled.

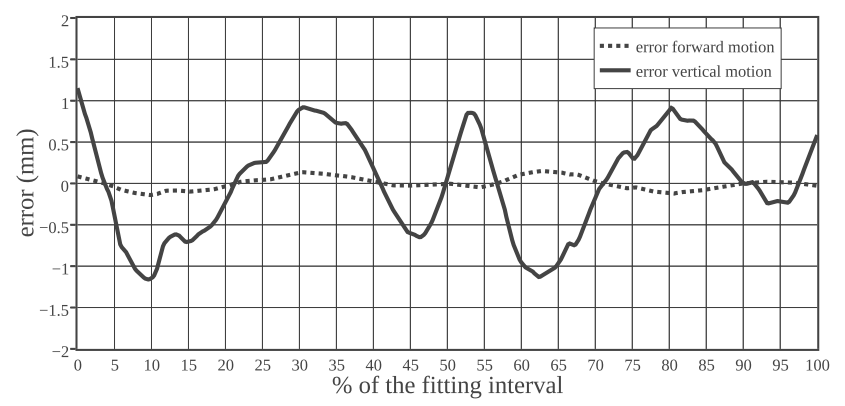

(b) Reconstruction error between the CoM trajectory and its fitting with the proposed model. Over the whole cycle, the reconstruction error remains below $1.5 \mathrm{~mm}$ and it is mainly contained in the vertical direction.

Figure 5: Illustration of the reconstruction of the center of mass trajectory (a) and its reconstruction error (b) during one stride.

correlate with the height of the subjects. Fig. 7 highlights the linear correlation between the radius parameters $R$ and $r$ of the cycloid and the height of the subjects. For both correlations, the computed p-value is lower than 0.01 and the coefficient of correlations is 0.67 and 0.61 respectively.

Fig 8 shows the evolution of the mean value of the

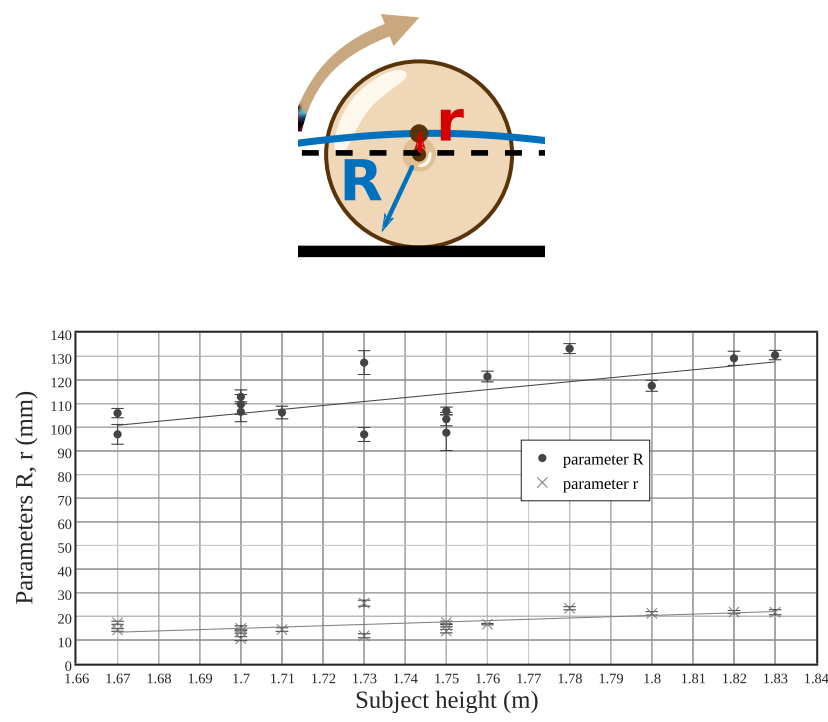

Figure 7: On top, the scheme of the wheel with the notations of the model: $R$ is the radius of the wheel while $r$ is the distance of the point to the wheel center. Bellow, a scatter plot showing the evolution of the mean radius parameters $R$ and $r$ according to the subject's sizes. The standard deviation of the parameters is low (below $5 \mathrm{~mm}$ ) for all the subjects. It appears that these two parameters are correlated to size of the subjects.

parameter $z_{0}$ (the height of the wheel) according to the height of the subjects. Unsurprisingly, a strong correlation is observed with $p \leq 0.001$ and a correlation coefficient with value 0.87 .

Finally, Fig 9a highlights the good affine approximation of the wheel angle $\theta$ according to time. For its part, Fig $9 \mathrm{~b}$ shows the mean and the standard deviation of the angular velocity of the cycloid according to the subject heights. It highlights the weak correlation $(p \geq 0.83)$ between the angular velocity of the cycloid and the size of the subjects. 


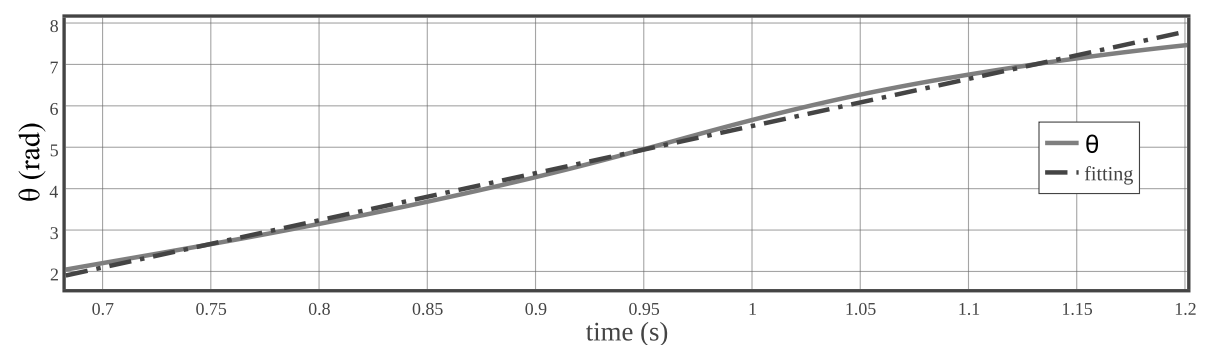

(a) Evolution of $\theta$ according to time and the corresponding linear fitting. It follows that $\theta$ can be well approximated by an affine function of time represented by Eq. (4).

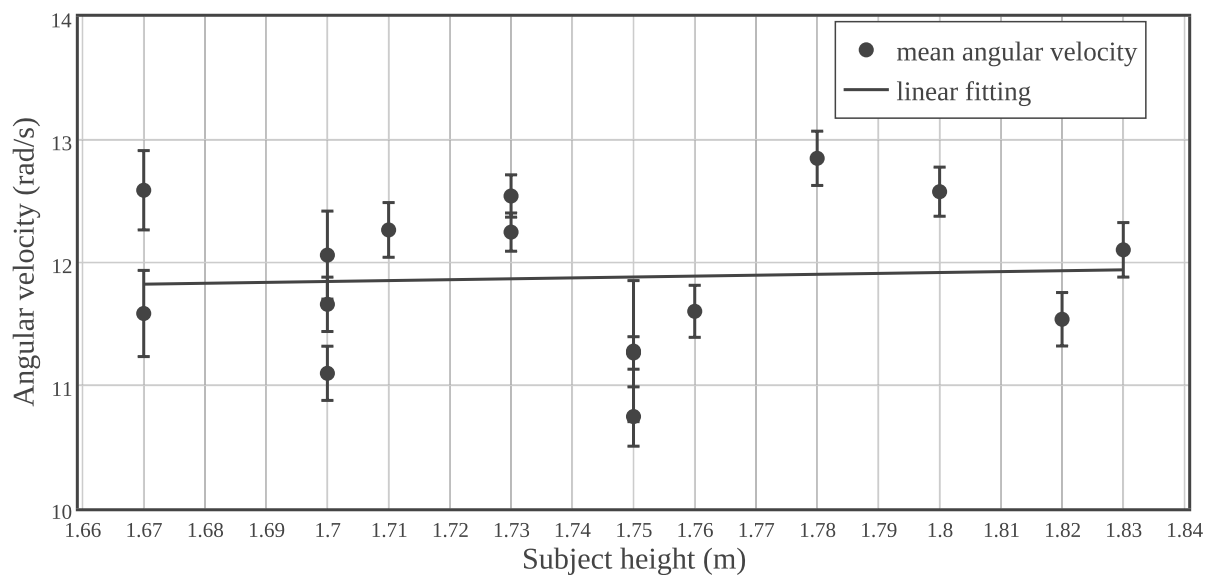

(b) Variation of the mean angular velocity $\omega$ of the cycloid according to the subjects' size.

Figure 9: Results of the fitting of $\theta$ with an affine approximation (a) and evolution of the mean angular velocity regarding to the subject height (b).

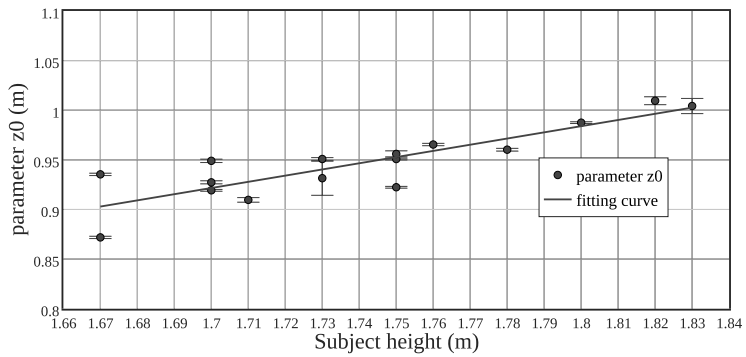

Figure 8: Evolution of the mean altitude $z_{0}$ according to the subject's size. The standard deviation of this parameter for each subject is very weak (below $2 \mathrm{~mm}$ ). Furthermore, the altitude is strongly correlated to the size of the subjects $p \leq 0.001$ with a correlation coefficient of 0.87 .

\subsection{The segmentation is embedded in the model}

The gait cycle has a natural segmentation due to the transitions between single and double support phases, and the extraction of this segmentation is relatively easy for the case of our model. The curtate cycloid has a specific shape with one minimum and two crossing points with the horizontal axis during one period. If we look at the two time instants where the cycloid crosses the horizontal axis at level $z 0$, they approximatively match the time of start and end of the double support phase respectively. this observation is assessed by Fig. 10 which shows the mean prediction error of the start and end time instants of the double support phases for all the subjects. We can observe that in average the two instants are well captured by the model.

\section{Discussions}

Our study shows that the center of mass of a walker follows the trajectory of a point attached to a virtual wheel moving on a horizontal plane at a constant velocity.

\subsection{Accuracy of the model}

The most important feature of our model is that the level of error in the vertical motion shown in Fig. 6 is one order of magnitude less than the existing models in the literature ${ }^{[21,22,23]}$, which overestimate the vertical position by up to $2 \mathrm{~cm}$.

In fact, our model fits the reconstructed trajectories with a higher precision than the accuracy of the measurement systems which is around $2 \mathrm{~mm}$ with classic and accurate motion capture systems ${ }^{[24]}$. In other words, it is not possible to go beyond this quality of fitting for this state-of-the-art measurement system. Moreover, even using force and moment sensors, the observability conditions of the center of mass are very weak along the direction of 


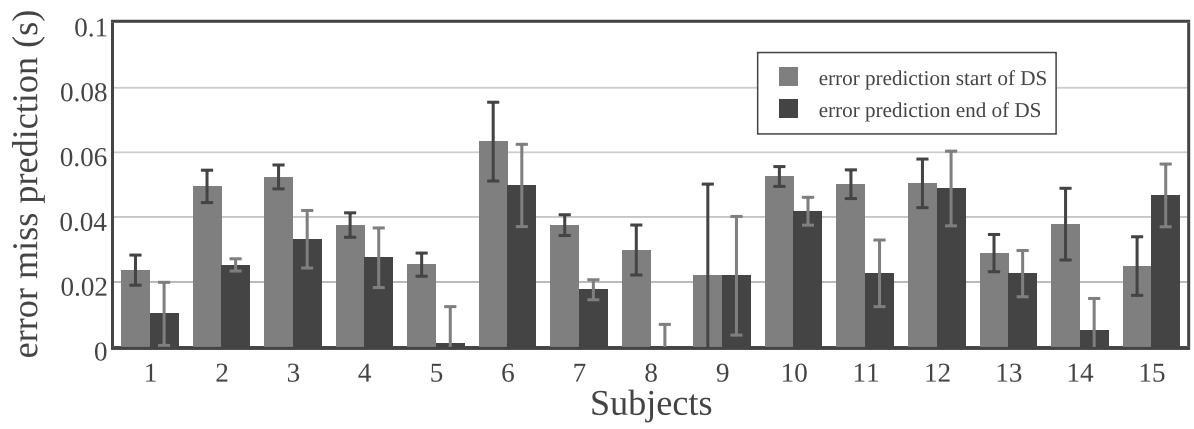

Figure 10: Bar graph of the prediction error of the time instants of start and end of the double support phases. In average, the two instants defining the double support are well captured by the model with only few milliseconds of errors.

the contact forces ${ }^{[17]}$, which leads mostly to CoM height misestimation.

\subsection{An intuitive model with few parameters}

Only three parameters are necessary to describe all the CoM trajectory with our model. This can to be compared to the other models in the literature ${ }^{[21]}$, where the models are composed of pendulum and inverted pendulum, but they necessarily need more parameters, for instance the location of the pendulum pivot points.

It is worth to emphasize that the few number of the parameters of our model keeps a simple intuitive geometrical interpretation, in contrast with possible purely numeric parametrization such as Fourier transforms or other approaches based on moments or frequency-domain representations ${ }^{[25]}$.

\subsection{A stable descriptor and reliable predictor}

Our model allows to study both a single step and an average steady gait. Indeed, the parameters can be identified from one single step, and our experimental results show that these parameters have relatively low variability regarding the variability of the gait: every subject samples their parameters following a tight probability distribution which spans a space comparable to the variability of the gait itself. Therefore, this also turns our model into a reliable predictor for the motion of the center of mass, given this distribution.

\subsection{A segmentation-free model}

To the best of our knowledge, our model is the first time-domain model which is free of segmentation. In other words, the curtate cycloid is a single curve. It is not composed of various patterns defined on multiple intervals. The model does not require any distinction between single and double support phases. ${ }^{2}$

More than that, the model contains the segmentation of the locomotion. Indeed, the minima of the cycloidal

\footnotetext{
2 The rolling of an egg on a table has already been considered as model to explain the shape of the center of mass trajectory during walking ${ }^{[26]}$. To the best of author's knowledge, no results have confirmed yet this hypothesis on a population of individuals.
}

trajectory and its crossing with the wheel axis define together the beginning and the end of the double support.

This gives an interesting echo to the observation made by Sternad and Schaal that "Segmentation of endpoint trajectories does not imply segmented control" ${ }^{[27]}$. This suggests that a possible continuous control of the CoM position can be achieved using this kind of models.

\subsection{A useful model for walking gait analysis}

The 3-dimensional space defining the curtate cycloids allows to study walking gait in a simple way. The correlations between these parameters and the height of the subjects are easy to capture. It is then possible to make use of this model to study other features such as the effect of sensory-motor impairments on walking motion generation.

Finally, one striking feature of our model is that it creates also naturally another moving reference frame which is the center of the virtual wheel. In this reference frame the CoM produces orbiting trajectories at a constant distance from the origin, which hovers in the space at constant height and low-varying velocity. The center of the wheel produces then smooth and regular locomotor trajectories, easy to exploit to study broader properties of walking motion generation.

\subsection{Limitations of the model}

While our model fits well with a population of 16 people, it is only applicable in the context of nominal walk. It has already been observed in the literature ${ }^{[30]}$ that during running, the CoM follows a different shape than the one proposed. An interesting study would consist of studying the existence of similar geometric model in the context of running motions. This would lead to another analytic study on the switching transition between walking and running which remains an open question.

\section{Conclusion: a robotics perspective}

In this work, we have experimentally shown that the centre of mass trajectory follows a curtate cycloid during normal walking. The proposed idea offers new features compared to the current literature: it only depends on 3 parameters varying according to the subject size; it is a segmentation-free model and the prediction accuracy 


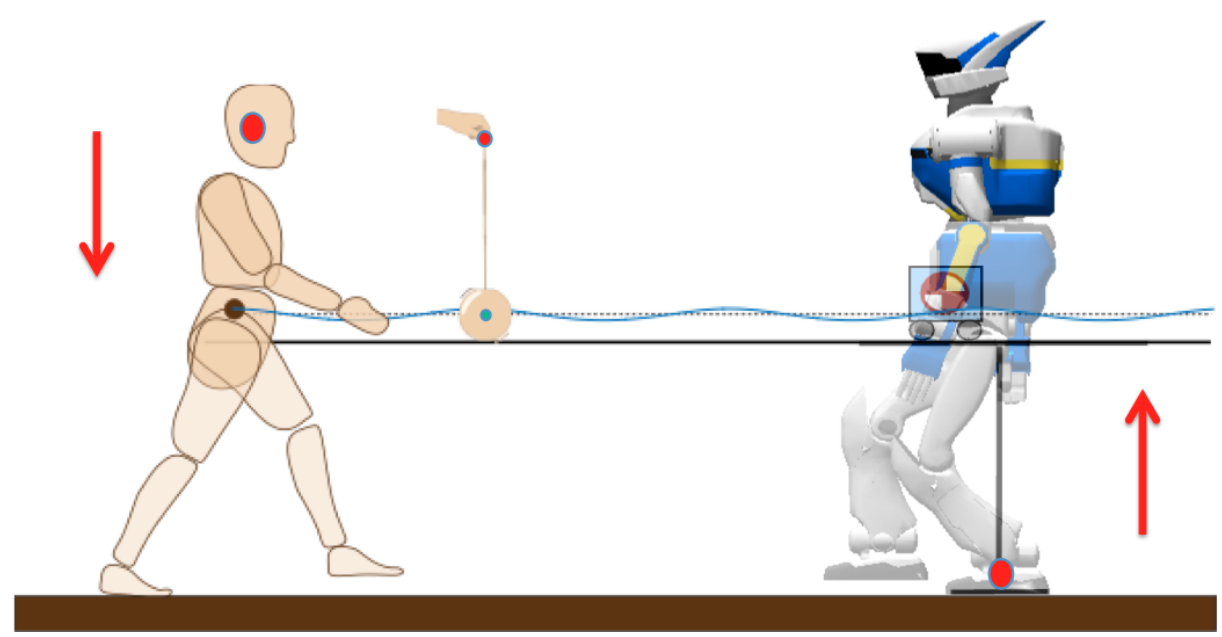

Figure 11: The Yoyo-Man model opens promising research routes to continue exploring the computational foundations of human and humanoid walking. Most existing walking controllers for humanoid robots consider a bottom-up approach based on the control of the so-called Zero Moment Point (ZMP) ${ }^{[28,29]}$. With the Yoyo-Man model, we suggest new plausible walking bottom-up control schemes that benefit from the knowledge of the Centre of Mass motion.

exceeds existing approaches.

This work is part of the ERC Actanthrope project developed in the Gepetto team at LAAS-CNRS ${ }^{3}$. Actanthrope promotes a neuro-robotics perspective to explore original models of anthropomorphic action. The project targets contributions to humanoid robot autonomy, to advanced human body simulation, and to a new theory of embodied intelligence by promoting a motion based semiotics of the human action. The results presented in this paper contributes to the so-called Yoyo-Man model introduced in ${ }^{[31]}$. The objective of this model is to propose an alternative approach to the control of humanoid robots (Fig. 11). This work may also inspire the design of new bipedal walkers $[32,33]$ by exploiting locomotion synergies to simplify both the architecture and the control of such robots.

\section{Acknowledgements}

We first thank the volunteers who took part in the experiment. We warmly thank Bruno Watier from LAAS-CNRS and University of Toulouse for helping us in the acquisition of motion capture data.

This work is supported by the European Research Council through the Actanthrope project and the French National Research Agency project Loco3D.

\section{References}

[1] M. W. Whittle, Gait analysis: an introduction. Butterworth-Heinemann, 2014.

[2] R. M. Alexander, "Walking made simple," Science, vol. 308 , no. 5718, pp. 58-59, 2005.

[3] M. L. Latash and V. Zatsiorsky, Biomechanics and Motor Control: Defining Central Concepts. Academic Press, 2015.

[4] V. T. Inman, H. D. Eberhart, et al., "The major determinants in normal and pathological gait," J Bone Joint Surg Am, vol. 35, no. 3, pp. 543-558, 1953.

[5] U. Della Croce, P. O. Riley, J. L. Lelas, and D. C. Kerrigan, "A refined view of the determinants of gait," Gait \& posture, vol. 14, no. 2, pp. 79-84, 2001.

[6] G. Cavagna and R. Margaria, "Mechanics of walking.," Journal of Applied Physiology, vol. 21, no. 1, pp. 271-278, 1966.

[7] A. D. Kuo, "The six determinants of gait and the inverted pendulum analogy: A dynamic walking perspective," Human movement science, vol. 26, no. 4, pp. 617-656, 2007.

[8] M. W. Whittle, "Three-dimensional motion of the center of gravity of the body during walking," Human Movement Science, vol. 16, no. 2, pp. 347-355, 1997.

[9] C. R. Lee and C. T. Farley, "Determinants of the center of mass trajectory in human walking and running.," Journal of experimental biology, vol. 201, no. 21, pp. 2935-2944, 1998.

[10] L. Tesio, V. Rota, C. Chessa, and L. Perucca, "The $3 \mathrm{~d}$ path of body centre of mass during adult human walking on force treadmill," Journal of biomechanics, vol. 43, no. 5, pp. 938-944, 2010.

\footnotetext{
${ }^{3}$ https://actanthrope.laas.fr
} 
[11] G. Wu, S. Siegler, P. Allard, C. Kirtley, A. Leardini, D. Rosenbaum, M. Whittle, D. D. D'Lima, L. Cristofolini, H. Witte, O. Schmid, and I. Stokes, "ISB recommendation on definitions of joint coordinate system of various joints for the reporting of human joint motion-part I: ankle, hip, and spine. International Society of Biomechanics.," Journal of biomechanics, vol. 35, no. 4, pp. 543-548, 2002.

[12] G. Wu, F. C. T. Van Der Helm, H. E. J. Veeger, M. Makhsous, P. Van Roy, C. Anglin, J. Nagels, A. R. Karduna, K. McQuade, X. Wang, F. W. Werner, and B. Buchholz, "ISB recommendation on definitions of joint coordinate systems of various joints for the reporting of human joint motion - Part II: Shoulder, elbow, wrist and hand," Journal of Biomechanics, vol. 38, no. 5, pp. 981-992, 2005.

[13] P. De Leva, "Adjustments to Zatsiorsky-Seluyanov's segment inertia parameters," Journal of Biomechanics, vol. 29, no. 9, pp. 1223-1230, 1996.

[14] T. Shimba, "An estimation of center of gravity from force platform data," Journal of Biomechanics, vol. 17, no. 1, pp. 53-60, 1984.

[15] O. Caron, B. Faure, and Y. Brenière, "Estimating the centre of gravity of the body on the basis of the centre of pressure in standing posture," Journal of biomechanics, vol. 30, no. 11, pp. 1169-1171, 1997.

[16] F. Barbier, P. Allard, K. Guelton, B. Colobert, and A.-P. Godillon-Maquinghen, "Estimation of the 3-d center of mass excursion from force-plate data during standing," IEEE Transactions on neural systems and rehabilitation engineering, vol. 11, no. 1, pp. 31-37, 2003.

[17] J. Carpentier, M. Benallegue, N. Mansard, and J.-P. Laumond, "Center-of-mass estimation for a polyarticulated system in contact - A spectral approach," IEEE Transactions on Robotics, vol. 32, pp. 810-822, Aug 2016.

[18] T. Shimba, "An estimation of center of gravity from force platform data.," Journal of biomechanics, vol. 17, no. 1, pp. 53-60, 1984.

[19] S. a. Gard, S. C. Miff, and A. D. Kuo, "Comparison of kinematic and kinetic methods for computing the vertical motion of the body center of mass during walking," Human Movement Science, vol. 22, no. 6, pp. 597-610, 2004.

[20] R. Dumas, L. Chèze, and J.-P. Verriest, "Adjustments to McConville et al. and Young et al. body segment inertial parameters.," Journal of biomechanics, vol. 40, pp. 543-53, jan 2007.

[21] C. Hayot, S. Sakka, V. Fohanno, and P. Lacouture, "Biomechanical modeling of the $3 \mathrm{~d}$ center of mass trajectory during walking," Movement \& Sport Sciences, no. 4, pp. 99-109, 2016.
[22] W. Zijlstra and A. L. Hof, "Displacement of the pelvis during human walking: experimental data and model predictions," Gait $\&$ posture, vol. 6, no. 3, pp. 249-262, 1997.

[23] S. Sakka, C. Hayot, and P. Lacouture, "A generalized $3 \mathrm{~d}$ inverted pendulum model to represent human normal walking," in 2010 10th IEEE-RAS International Conference on Humanoid Robots, pp. 486-491, IEEE, 2010.

[24] D. A. Winter, Biomechanics and motor control of human movement. John Wiley \& Sons, 2009.

[25] A. E. Minetti, C. Cisotti, and O. S. Mian, "The mathematical description of the body centre of mass 3d path in human and animal locomotion," Journal of biomechanics, vol. 44, no. 8, pp. 1471-1477, 2011.

[26] B. M. Nigg, B. R. MacIntosh, and J. Mester, Biomechanics and biology of movement. Human Kinetics, 2000.

[27] D. Sternad and S. Schaal, "Segmentation of endpoint trajectories does not imply segmented control.," Experimental brain research, vol. 124 1, pp. 118-36, 1999.

[28] M. Vukobratović and B. Borovac, "Zero-moment point-thirty five years of its life," International Journal of Humanoid Robotics, vol. 1, no. 01, pp. 157-173, 2004.

[29] S. Kajita, F. Kanehiro, K. Kaneko, K. Fujiwara, K. Harada, K. Yokoi, and H. Hirukawa, "Biped walking pattern generation by using preview control of zero-moment point," in Robotics and Automation, 2003. Proceedings. ICRA'03. IEEE International Conference on, vol. 2, pp. 1620-1626, IEEE, 2003.

[30] C. R. Lee and C. T. Farley, "Determinants of the center of mass trajectory in human walking and running.," Journal of Experimental Biology, vol. 201, no. 21, pp. 2935-2944, 1998.

[31] J.-P. Laumond, M. Benallegue, J. Carpentier, and A. Berthoz, "The yoyo-man," To appear in the International Journal of Robotics Research, 2017.

[32] H. S. Jo and N. Mir-Nasiri, "Development of minimalist bipedal walking robot with flexible ankle and split-mass balancing systems," International Journal of Automation and Computing, vol. 10, no. 5, pp. 425-437, 2013.

[33] G. Saurel, J. Carpentier, N. Mansard, and J.-P. Laumond, "A simulation framework for simultaneous design and control of passivity based walkers," in 2016 IEEE International Conference on Simulation, Modeling, and Programming for Autonomous Robots SIMPAR, 2016. 


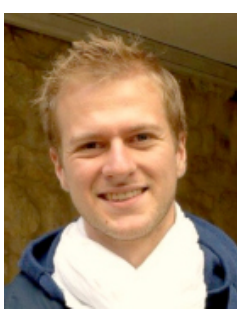

Justin Carpentier received the MSc in Applied Mathematics and Computer Science from ENS Paris-Saclay, France in 2013. In 2014, he was a visiting student inside the Optimization, Robotics and Biomechanics group at University of Heidelberg, Germany. Since 2014, he has been a $\mathrm{PhD}$ candidate inside the Gepetto team at Laboratory for Analysis and Architecture of Systems, Toulouse, France. His research interests include the motion generation for humanoid robots, bipedal locomotion and numerical optimal control.

email: justin.carpentier@laas.fr (Corresponding author)

ORCID Id: 0000-0001-6585-2894

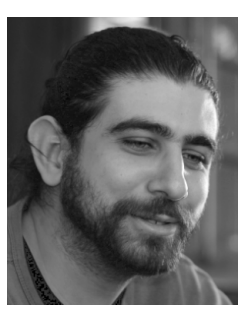

Mehdi Benallegue received the engineer degree from the National Institute of Computer Science (INI), Algeria, in 2007, the M.Sc. degree from the University of Paris 7, Paris, France, in 2008, and the Ph.D. degree from the University of Montpellier 2, France, in 2011. Previously, he was a Research Associate with the Laboratory for Analysis and Architecture of Systems, Toulouse, France. He is now with the Humanoid Research Group, at the National Institute of Advanced Industrial Science and Technology, Tsukuba, Japan. His research interests include humanoid robotics, state observers, locomotion, imitation, learning, and computational geometry.

email: mehdi@benallegue.com

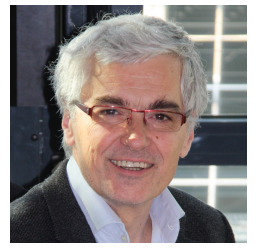

Jean-Paul Laumond is Research Director at LAAS-CNRS in Toulouse, France. He is a member of the French Academy of Technology. His research is devoted to robot motion. In the 90's, he has been the coordinator of two European Esprit projects, both dedicated to robot motion planning and control. In the early 2000's he created and managed Kineo CAM, a spin-off company from LAAS-CNRS devoted to develop and market motion planning technology. Siemens acquired Kineo CAM in 2012. In 2006, he launched the research team Gepetto dedicated to Human Motion studies. He teaches Robotics at Ecole Normale Suprieure in Paris. He has published more than 150 papers in international journals and conferences in Robotics, Computer Science, Automatic Control and recently in Neurosciences. He has been the 2011-2012 recipient of the Chaire Innovation Technologique Liliane Bettencourt at Collège de France in Paris. His current project Actanthrope is devoted to the computational foundations of anthropomorphic action and it is supported by the European Research Council (ERC-ADG 340050). His research interests include humanoid robotics, human locomotion, digital actor animation and motion planning.

email: jpl@laas.fr 